\title{
Use of an Automated Bolus Calculator Reduces Fear of Hypoglycemia and Improves Confidence in Dosage Accuracy in Patients with Type 1 Diabetes Mellitus Treated with Multiple Daily Insulin Injections
}

\author{
Katharine Barnard, Ph.D., C.Psychol., ${ }^{1}$ Christopher Parkin, M.S., ${ }^{2}$ Amanda Young, M.Sc., ${ }^{1}$ \\ and Mansoor Ashraf, M.B.B.S. ${ }^{3}$
}

\section{Abstract}

\section{Background:}

Many patients do not intensify their insulin regimens. It is believed that lack of adherence may be largely due to fear of hypoglycemia. We hypothesized that utilization of an automated bolus calculator (bolus advisor) might reduce fear of hypoglycemia and encourage patients to achieve improved glycemic control.

\section{Method:}

We surveyed 1,412 type 1 diabetes mellitus (T1DM) patients treated with multiple daily insulin injection therapy at 270 hospitals in the United Kingdom and Republic of Ireland to assess their attitudes and behaviors regarding insulin therapy after use of a bolus advisor (Accu-Chek ${ }^{\circledR}$ Aviva Expert blood glucose meter and bolus advisor system, Roche Diagnostics). The device automatically calculates bolus dosages based on current blood glucose values, anticipated meal intake, and other parameters.

\section{Results:}

Five hundred eighty-eight T1DM patients responded to the survey. Respondents were predominantly female, age $<1$ to 70 years, with diabetes duration of $<1$ to $>15$ years. Respondents had 4-12 weeks prior experience using the bolus advisor. $76.7 \%$ of respondents indicated current bolus advisor use to calculate insulin boluses for meals/snacks always or quite often. 52.0\% of respondents indicated that fear of hypoglycemia was reduced (39.0\%) or significantly reduced (13.0\%). 78.8\% indicated that confidence in the insulin dose calculation improved (50.8\%) or significantly improved (28.0\%). $89.3 \%$ indicated that the bolus advisor made bolus calculation easy or very easy compared with manual calculation.

\section{Conclusions:}

Most patients felt that using the bolus advisor was easier than manual bolus calculation, improved their confidence in the accuracy of their bolus dosage, and reduced their fear of hypoglycemia. Randomized trials are needed to confirm these perceptions and determine whether bolus advisor use improves clinical outcomes.

J Diabetes Sci Technol 2012;6(1):144-149

Author Affiliations: ${ }^{1}$ University of Southampton, Alpha House, Southampton Science Park, Chilworth, Southampton, United Kingdom; ${ }^{2}$ CGParkin Communications, Inc., Las Vegas, Nevada; and ${ }^{3}$ Roche Diagnostics Limited, West Sussex, United Kingdom

Abbreviations: (ABC) automated bolus calculator, (BG) blood glucose, (CHO) carbohydrate, (HbA1c) hemoglobin A1c, (I:CHO) insulin-tocarbohydrate ratio, (MDI) multiple daily injection, (SMBG) self-monitoring of blood glucose, (T1DM) type 1 diabetes mellitus

Keywords: bolus, bolus calculator, diabetes, insulin, multiple daily injection, type 1 diabetes

Corresponding Author: Christopher Parkin, M.S., CGParkin Communications, Inc., 3722 Las Vegas Boulevard South, Suite 2402, Las Vegas, NV 89158; email address chris@cgparkin.org 


\section{Introduction}

L controlled clinical trials have shown that intensive management of glycemia and other risk factors associated with diabetes can significantly decrease the development and/or progression of microvascular and macrovascular complications. $^{1-5}$ Despite advances in developing new medications, insulin delivery systems, and glucose monitoring technology, a significant percentage of patients with diabetes remain well above their glycemic goals. Data from the most recent United Kingdom National Health Service audit showed that over $70 \%$ of adults with type 1 diabetes (T1DM) were above their glycemic targets; approximately 33\% were in very poor control with hemoglobin A1c (HbA1c) levels >10.0\%. ${ }^{6}$ These findings are similar to those reported in 2006-07 and 2007-08, reflecting persistent poor control.

The roots of poor diabetes control often begin in childhood with persistent poor control continuing into adulthood. Pediatric Scottish audit data showed that only $10 \%$ of children were meeting the National Institute for Health and Clinical Excellence guideline target for blood glucose (BG) control. ${ }^{7}$ Similar data from England and Wales showed that $81 \%$ of children $<1$ to 11 years of age fail to achieve a target HbA1c level $<7.5 \%$; $28 \%$ of children under 12 years old have $\mathrm{HbA1c}$ levels $>9.5 \%{ }^{6}$

Achieving optimal glycemic control requires a high level of daily self-management. For patients with T1DM, this often includes intensive insulin therapy with dose adjustment based upon carbohydrate intake and activity, and frequent glucose monitoring. ${ }^{1,8}$ Despite the proven benefits of effective diabetes management, many people with diabetes are reluctant or unable to follow and/ or adjust their insulin regimens as needed. ${ }^{9-11}$ It has also been shown that many people with T1DM perform self-monitoring of blood glucose (SMBG) at suboptimal levels. ${ }^{12-14}$

Because diabetes is largely a self-managed disease with a significant psychosocial impact on patients and their families, ${ }^{15}$ many people with diabetes experience poorer quality of life than their nondiabetic counterparts. ${ }^{16}$ This may lead to less rigorous glucose self-monitoring or insulin adjustment, which in turn may result in increased glucose variability. The burden of diabetes self-management may partly explain why most people with diabetes do not achieve optimal glycemic control; however, another compounding factor may be an underlying fear of hypoglycemia.
Hypoglycemia is a common consequence of intensive insulin management. ${ }^{17}$ Patients with T1DM experience an average of two episodes of symptomatic hypoglycemia each week and at least one episode of severe, disabling hypoglycemia annually. ${ }^{18}$ Significant hypoglycemia, frequently accompanied by substantial physical, cognitive, and emotional disturbance, is a major cause of morbidity and mortality. ${ }^{19}$

People with diabetes "learn quickly that hypoglycaemic episodes are physically aversive, potentially dangerous and a source of possible social embarrassment." 20 A large, study by Anderbro and colleagues identified frequency of severe hypoglycemia as the most significant factor associated with fear of hypoglycemia in adults with T1DM. ${ }^{21}$ This finding was particularly significant among women. ${ }^{21}$ Thus, fear of hypoglycemia often becomes a key obstacle to intensifying therapy and/or adhering to prescribed insulin regimens, ${ }^{22-24}$ which often leads to poor metabolic control and subsequent health outcomes. ${ }^{25}$

Compounding the problem are the challenges that arise when clinicians escalate patients to multiple daily injection (MDI) therapy to provide better glycemic control. Calculation of an insulin dose is a complex process in which numerous factors, such as preprandial glucose level, grams of carbohydrate $(\mathrm{CHO})$, insulin sensitivity, insulinto- $\mathrm{CHO}$ ratio (I:CHO), and active insulin on board should be taken into account. Because manual calculation of insulin boluses is complex and time-consuming, patients may rely on empirical estimates, which may result in hypoglycemia.

New technology provides the capability to automatically calculate bolus insulin dosages to cover $\mathrm{CHO}$ intake and address out-of-range BG levels. We hypothesized that utilization of an automated bolus calculator (ABC) (bolus advisor) might reduce fear of hypoglycemia and encourage patients to achieve improved glycemic control. To test this hypothesis, we surveyed T1DM patients who had recently begun using a bolus advisor as a component of their MDI therapy.

\section{Method}

In this multicenter survey, we sent a 45-item questionnaire to 1,412 T1DM patients treated with MDI therapy. The survey was conducted from April 2010 to January 2011. Subjects were drawn from 270 hospitals and clinics 
throughout the United Kingdom and Republic of Ireland. Subjects were required to have a minimum of 4 weeks of experience with the bolus advisor. There were no additional inclusion or exclusion criteria for subject selection.

The questionnaire was designed to obtain demographic data and information about user experiences with a bolus advisor (ACCU-CHEK ${ }^{\circledR}$ Aviva Expert BG meter and bolus advisor system, Roche Diagnostics, Indianapolis, IN). The bolus advisor system incorporates a bolus advisor with an integrated BG meter. Users can receive meal and correction bolus recommendations based upon a current BG value, planned $\mathrm{CHO}$ intake, and patient-specific therapy parameters stored in the device. The device automatically calculates the appropriate bolus for the user and stores BG and meal information in an electronic diary. A study by Zisser and colleagues demonstrated that the bolus advisor used by the surveyed patients improves postprandial glucose control without increasing the frequency of hypoglycemia. ${ }^{26}$ For our analysis, we used selected items from the questionnaire to assess user attitudes and behaviors regarding insulin therapy after using the bolus advisor as part of their MDI therapy.

Summary and descriptive statistics were produced. Free text responses were independently analyzed and thematically coded by two researchers. Interrater reliability was 93.85\% with consensus reached on disagreements by discussion.

\section{Results}

A total of 588 T1DM patients responded to the survey, a response rate of $41.6 \%$. Respondents were predominantly female, age $<1$ to 70 years, with diabetes duration ranging from $<1$ to $>15$ years (Table 1). Prior to completing the questionnaire, respondents had 4-12 weeks of experience using the bolus advisor.

More than 95\% $(n=560)$ of respondents indicated that they used MDI therapy with a long-acting analog. Results showed that $45.7 \%(n=264)$ of respondents had completed the National Health Service course on diabetes self-management; however, 92.9\% $(n=513)$ stated that they had been trained to count carbohydrates and use that information to determine bolus insulin dosages at meal times, and $83.7 \%(n=492)$ stated that prior to utilizing the bolus advisor they actually used carbohydrate counting to adjust their insulin dosages. Although $69.1 \%(n=400)$ of respondents said that they had been adjusting their I:CHO and insulin sensitivity ratios, $23.4 \%(n=94)$ of respondents indicated that they did so without the assistance of their health care provider. More than $45 \%(n=257)$ said that they performed SMBG $4-5$ times per day; $23.4 \%(n=133)$ stated that they tested $>6$ times per day.

Almost $77 \%$ of respondents indicated that they now use the bolus advisor to calculate insulin boluses for meals/ snacks: always $(53.6 \%, n=301)$ or quite often $(23.1 \%, n=130)$. However, only $41.5 \%(n=232)$ of respondents stated that they used the bolus advisor to calculate correction boluses: always $(15.0 \%, n=84)$, quite often $(26.5 \%, n=148)$, sometimes $(36.3 \%, n=203)$, very occasionally $(17.7 \%$, $n=99)$, or never $(4.5 \%, n=25)$. Among those who did administer correction boluses $(n=535)$, there was significant improvement in their perceptions of ease of dosage calculation using the bolus advisor (Figure 1). We also saw significant improvements in respondents'

\begin{tabular}{|c|c|c|}
\hline & $n$ & $\%$ \\
\hline \multicolumn{3}{|l|}{ Age (years) } \\
\hline $\begin{array}{l}<18 \\
18-30 \\
31-40 \\
41-50 \\
51-60 \\
61-70\end{array}$ & $\begin{array}{c}182 \\
107 \\
120 \\
107 \\
46 \\
26 \\
\end{array}$ & $\begin{array}{c}31.0 \\
18.2 \\
20.4 \\
18.2 \\
7.8 \\
4.4\end{array}$ \\
\hline \multicolumn{3}{|l|}{ Gender } \\
\hline $\begin{array}{l}\text { Male } \\
\text { Female }\end{array}$ & $\begin{array}{l}250 \\
338 \\
\end{array}$ & $\begin{array}{l}42.5 \\
57.5 \\
\end{array}$ \\
\hline \multicolumn{3}{|c|}{ Diabetes Duration (years) } \\
\hline $\begin{array}{l}<1-4 \\
5-10 \\
11-14 \\
\geq 15\end{array}$ & $\begin{array}{c}169 \\
130 \\
56 \\
233\end{array}$ & $\begin{array}{c}28.7 \\
22.1 \\
9.5 \\
39.6\end{array}$ \\
\hline
\end{tabular}

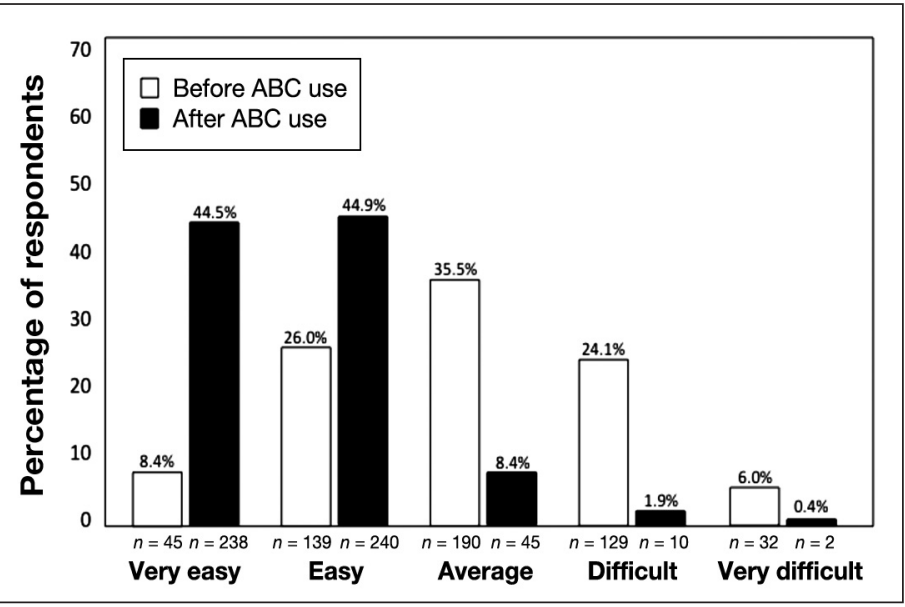

Figure 1. Perceived ease of calculating a correction bolus. 
perceptions of several diabetes management-related factors, including fear of hypoglycemia; confidence in insulin dosage calculations; ease of dosage calculation; ability to act upon SMBG results; BG control; ability to achieve glycemic goals; flexibility in meals, exercise, and lifestyle; and overall well-being (Table 2). Self-monitoring of BG frequency also increased after using the bolus advisor (Figure 2).

Out of 588 respondents, 520 provided general comments on their experiences with the bolus advisor; 715 responses were recorded. From these, four main themes were identified regarding diabetes control, impact on lifestyle, fear of hypoglycemia, and self-efficacy.

Regarding the impact of bolus advisor use on diabetes management, 81 respondents identified improved diabetes control as a benefit of using the bolus advisor. Reductions in $\mathrm{HbA1c}$ levels were reported as well as more general improvements in diabetes control:

"HbA1c is down from 10.9 to $8.3 \mathrm{mmol} /$ liter and is a great tool for my driving ability and convenience."

"It has changed my life, HbA1c is 8 from 12."

Sixty-eight respondents reported that use of the bolus advisor had a positive impact on their life overall and enabled them to minimize the burden of diabetes somewhat:

"It has made me a healthier person and made living with diabetes a lot easier."

"Makes me feel more secure in my control."

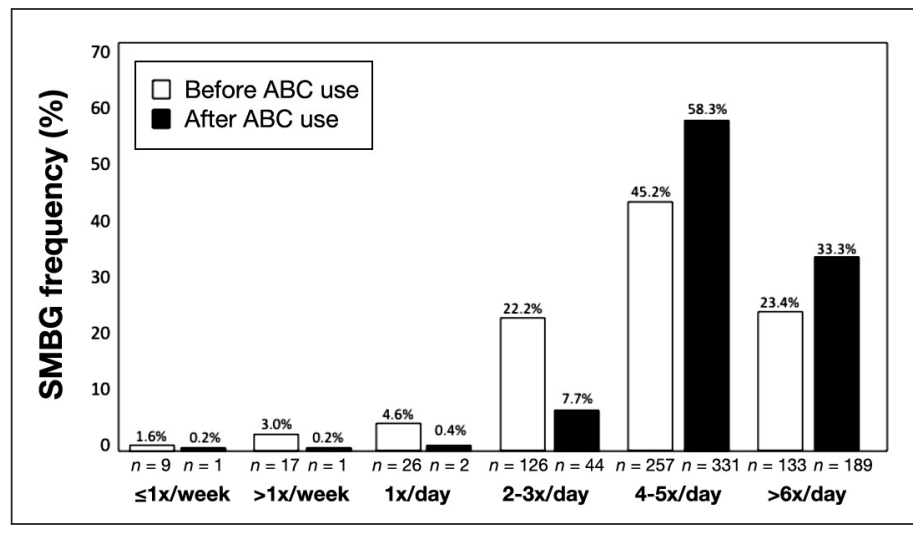

Figure 2. Change in SMBG frequency.

Although fear of hypoglycemia was not identified as a theme in itself, consistent with the quantitative statistical results, this issue was raised by a number of people under the guise of increased confidence and self-efficacy; 58 participants cited increased self-efficacy as a benefit of using the bolus advisor:

"I have much more confidence now and don't fear hypos because of it."

"I feel more confident about the insulin dose I have to take when eating."

"It has given us the confidence to move to MDI and made the transition an awful lot easier."

\section{Conclusions}

Intensive self-management of diabetes is complex and time-consuming, and creates a significant psychosocial burden on patients and their families. ${ }^{15}$ Adding to the

Table 2.

Perceived Improvement in Diabetes Management-Related Factors

\begin{tabular}{|l|c|c|c|c|c|} 
& $\begin{array}{c}\text { Significantly } \\
\text { improved }\end{array}$ & Improved & No change & Worsened \\
\hline Fear of hypoglycemia & $13.0 \%(73)$ & $39.0 \%(219)$ & $43.0 \%(241)$ & $4.8 \%(27)$ & $0.2 \%(1)$ \\
\hline Confidence in calculation & $28.0 \%(157)$ & $50.8 \%(285)$ & $16.8 \%(94)$ & $3.9 \%(22)$ & $0.5 \%(3)$ \\
\hline Ease of calculating bolus & $43.7 \%(245)$ & $41.2 \%(231)$ & $13.2 \%(74)$ & $1.8 \%(10)$ & $0.2 \%(1)$ \\
\hline Acting on SMBG results & $27.1 \%(152)$ & $54.2 \%(304)$ & $16.9 \%(95)$ & $1.8 \%(10)$ & $0.0 \%(0)$ \\
\hline Control of BG levels & $20.1 \%(113)$ & $53.5 \%(300)$ & $23.0 \%(129)$ & $3.2 \%(18)$ & $0.2 \%(1)$ \\
\hline Ability to achieve BG goals & $13.4 \%(75)$ & $53.7 \%(301)$ & $30.8 \%(173)$ & $2.0 \%(11)$ & $0.2 \%(1)$ \\
\hline Flexibility in lifestyle & $20.5 \%(115)$ & $42.4 \%(238)$ & $35.3 \%(198)$ & $1.8 \%(10)$ & $0.0 \%(0)$ \\
\hline Overall well-being & $17.5 \%(98)$ & $54.4 \%(305)$ & $26.7 \%(150)$ & $1.2 \%(7)$ & $0.2 \%(1)$ \\
\hline
\end{tabular}


burden of self-management is the underlying, debilitating fear of hypoglycemia, ${ }^{22-24}$ which can result in poor adherence to insulin regimens and subsequent poor metabolic control. ${ }^{25}$

Results from our survey suggest that use of a bolus advisor to aid in determining bolus insulin dosages may alleviate some of the fears and inconveniences associated with MDI therapy in people with T1DM. The majority of patients surveyed felt that using the bolus advisor was easier than manual bolus calculation. Moreover, we saw reduced fear of hypoglycemia, increased confidence in bolus calculation, improved ability to control BG levels and achieve glycemic goals, a sense of increased flexibility in lifestyle, and improvement in overall well-being.

It is noteworthy that greater than $80 \%(n=456)$ of respondents indicated improvement in their ability to act upon SMBG data. This perception is supported by the changes seen in SMBG frequency. After using the bolus advisor, the number of patients testing 4 to 5 times per day increased $28 \%$, from 257 to 331, whereas the number of patients testing $>6$ times per day increased more than $42 \%$, from 133 to 189 . This suggests that patients are, in fact, willing to perform SMBG at optimal frequencies when they see the purpose and value of their testing.

Given the benefits of tight metabolic control, it is critical that health care providers utilize available technologies that not only facilitate effective glucose management but also address their patients' concerns about safety and lifestyle. Although findings from our study strongly suggest that use of the bolus advisor positively addresses these concerns, randomized trials are needed to confirm these perceptions and determine whether bolus advisor use improves clinical outcomes.

\section{Disclosures:}

Funding for the survey was provided by Roche Diagnostics Limited, United Kingdom. Katharine Barnard has worked as a consultant for Novo Nordisk, Roche Diagnostics, and LifeScan. Christopher Parkin has worked as a consultant for Roche Diagnostics, DexCom, and Generex Pharmaceuticals. Mansoor Ashraf is an employee of Roche Diagnostics.

\section{References:}

1. Diabetes Control and Complications Trial Study Group. The effect of intensive treatment of diabetes on the development and progression of long-term complications in insulin-dependent diabetes mellitus. N Engl J Med. 1993;329(14):977-86.

2. UK Prospective Diabetes Study Group. Intensive blood-glucose control with sulphonylureas or insulin compared with conventional treatment and risk of complications in patients with type 2 diabetes (UKPDS 33). Lancet. 1998;352(9131):837-53.

3. Nathan DM, Cleary PA, Backlund JY, Genuth SM, Lachin JM, Orchard TJ, Raskin P, Zinman B; Diabetes Control and Complications Trial/Epidemiology of Diabetes Interventions and Complications (DCCT/EDIC) Study Research Group. Intensive diabetes treatment and cardiovascular disease in patients with type 1 diabetes. $\mathrm{N}$ Engl J Med. 2005;353(25):2643-53.

4. Holman RR, Paul SK, Bethel MA, Matthews DR, Neil HA. 10-year follow-up of intensive glucose control in type 2 diabetes. $\mathrm{N}$ Engl J Med. 2008;359(15):1577-89.

5. Gaede P, Lund-Andersen $\mathrm{H}$, Parving HH, Pedersen O. Effect of a multifactorial intervention on mortality in type 2 diabetes. $\mathrm{N}$ Engl J Med. 2008;358(6):580-91.

6. National Diabetes Audit Executive Summary 2008-2009. Key findings about the quality of care for people with diabetes in England and Wales. England: The NHS Information Centre; 2010.

7. Scottish Study Group for the Care of the Young with Diabetes. A longitudinal observational study of insulin therapy and glycaemic control in Scottish children with type 1 diabetes: DIABAUD 3. Diabet Med. 2006;23(11):1216-21.

8. Evans JM, Newton RW, Ruta DA, MacDonald TM, Stevenson RJ Morris AD. Frequency of blood glucose monitoring in relation to glycaemic control: observational study with diabetes database. BMJ. 1999;319(7202):83-6.

9. Di Battista AM, Hart TA, Greco L, Gloizer J. Type 1 diabetes among adolescents: reduced diabetes self-care caused by social fear and fear of hypoglycemia. Diabetes Educ. 2009;35(3):465-75.

10. Morris AD, Boyle DI, McMahon AD, Greene SA, MacDonald TM, Newton RW. Adherence to insulin treatment, glycaemic control, and ketoacidosis in insulin-dependent diabetes mellitus. The DARTS/ MEMO Collaboration. Diabetes Audit and Research in Tayside Scotland. Medicines Monitoring Unit. Lancet. 1997;350(9090):1505-10.

11. Smith CB, Choudhary P, Pernet A, Hopkins D, Amiel SA Hypoglycemia unawareness is associated with reduced adherence to therapeutic decisions in patients with type 1 diabetes: evidence from a clinical audit. Diabetes Care. 2009;32(7):1196-8.

12. Moreland EC, Volkening LK, Lawlor MT, Chalmers KA, Anderson B], Laffel LM. Use of a blood glucose monitoring manual to enhance monitoring adherence in adults with diabetes: a randomized controlled trial. Arch Intern Med. 2006;166(6):689-95.

13. Self-monitoring of blood glucose among adults with diabetes-United States, 1997-2006. MMWR Morb Mortal Wkly Rep. 2007;56(43):1133-7.

14. Brindisi MC, Hahn J, Chiasson JL, Rabasa-Lhoret R. Under-utilization of capillary glucose monitoring by type 2 diabetic patients. Diabetes Res Clin Pract. 2007;75(1):123-5.

15. Snoek FJ, Skinner TC. Psychological counselling in problematic diabetes: does it help? Diabet Med. 2002;19(4):265-73. 
16. Barnard KD, Speight J, Skinner TC. Quality of life and impact of continuous subcutaneous insulin infusion for children and their parents. Practical Diabetes International. 2008;25(7):278-83.

17. Cryer PE. Hypoglycemia: pathophysiology, diagnosis, and treatment. New York: Oxford University Press; 1997.

18. MacLeod KM, Hepburn DA, Frier BM. Frequency and morbidity of severe hypoglycaemia in insulin-treated diabetic patients. Diabet Med. 1993;10(3):238-45.

19. Gilbert RE, Fulcher G. Reduction in fear of hypoglycemia in subjects with sub-optimally controlled diabetes using a glargine insulin-based treatment regimen. Can J Diabetes. 2008;32(2):101-6.

20. Brouhard BH. Hypoglycemia. In: Travis LB, Brouhard BH, Schreiner BJ, editors. Diabetes mellitus in children and adolescents. Philadelphia: W.B. Saunders; 1987. p. 169-78.

21. Anderbro T, Amsberg S, Adamson U, Bolinder J, Lins PE, Wredling R, Moberg E, Lisspers J, Johansson UB. Fear of hypoglycaemia in adults with type 1 diabetes. Diabet Med. 2010;27(10):1151-8.

22. Cryer PE. Hypoglycemia: still the limiting factor in the glycemic management of diabetes. Endocr Pract. 2008;14(6):750-6.

23. Cryer PE. Diverse causes of hypoglycemia-associated autonomic failure in diabetes. N Engl J Med. 2004;350(22):2272-9.

24. Riddle MC. The underuse of insulin therapy in North America. Diabetes Metab Res Rev. 2002;18(Suppl 3):S42-S9.

25. Wild D, von Maltzahn R, Brohan E, Christensen T, Clauson P, Gonder-Frederick L. A critical review of the literature on fear of hypoglycemia in diabetes: implications for diabetes management and patient education. Patient Educ Couns. 2007;68(1):10-5.

26. Zisser H, Wagner R, Pleus S, Haug C, Jendrike N, Parkin C, Parkin C, Schweitzer M, Freckmann G. Clinical performance of three bolus calculators in subjects with type 1 diabetes mellitus: a headto-head-to-head comparison. Diabetes Technol Ther. 2010;12(12):955-61. 